\title{
Transcriptome analysis of Cymbidium sinense and its application to the identification of genes associated with floral development
}

\author{
Jianxia Zhang ${ }^{1,2}$, Kunlin $\mathrm{Wu}^{1}$, Songjun Zeng ${ }^{1}$, Jaime A Teixeira da Silva ${ }^{3}$, Xiaolan Zhao ${ }^{4}$, Chang-En Tian ${ }^{5}$,
} Haoqiang $\mathrm{Xia}^{6}$ and Jun Duan ${ }^{1 *}$

\begin{abstract}
Background: Cymbidium sinense belongs to the Orchidaceae, which is one of the most abundant angiosperm families. C. sinense, a high-grade traditional potted flower, is most prevalent in China and some Southeast Asian countries. The control of flowering time is a major bottleneck in the industrialized development of C. sinense. Little is known about the mechanisms responsible for floral development in this orchid. Moreover, genome references for entire transcriptome sequences do not currently exist for $C$. sinense. Thus, transcriptome and expression profiling data for this species are needed as an important resource to identify genes and to better understand the biological mechanisms of floral development in C. sinense.

Results: In this study, de novo transcriptome assembly and gene expression analysis using Illumina sequencing technology were performed. Transcriptome analysis assembles gene-related information related to vegetative and reproductive growth of $C$. sinense. Illumina sequencing generated 54,248,006 high quality reads that were assembled into 83,580 unigenes with an average sequence length of 612 base pairs, including 13,315 clusters and 70,265 singletons. A total of 41,687 (49.88\%) unique sequences were annotated, 23,092 of which were assigned to specific metabolic pathways by the Kyoto Encyclopedia of Genes and Genomes (KEGG). Gene Ontology (GO) analysis of the annotated unigenes revealed that the majority of sequenced genes were associated with metabolic and cellular processes, cell and cell parts, catalytic activity and binding. Furthermore, 120 flowering-associated unigenes, 73 MADS-box unigenes and 28 CONSTANS-LIKE (COL) unigenes were identified from our collection. In addition, three digital gene expression (DGE) libraries were constructed for the vegetative phase (VP), floral differentiation phase (FDP) and reproductive phase (RP). The specific expression of many genes in the three development phases was also identified. 32 genes among three sub-libraries with high differential expression were selected as candidates connected with flower development.
\end{abstract}

Conclusion: RNA-seq and DGE profiling data provided comprehensive gene expression information at the transcriptional level that could facilitate our understanding of the molecular mechanisms of floral development at three development phases of $C$. sinense. This data could be used as an important resource for investigating the genetics of the flowering pathway and various biological mechanisms in this orchid.

Keywords: Floral development, Flowering time, Digital gene expression, Transcriptome, Cymbidium sinense

\footnotetext{
*Correspondence: duanj@scbg.ac.cn

'Key Laboratory of South China Agricultural Plant Genetics and Breeding, South China Botanical Garden, The Chinese Academy of Sciences, Guangzhou 510650, China

Full list of author information is available at the end of the article
} 


\section{Background}

The Orchidaceae is one of the largest and most widespread families of flowering plants, with more than 250000 species [1]. Cymbidium, a very important economically flowering genus with the Orchidaceae, has nearly 55 species throughout the world and is widespread in tropical and subtropical Asia, reaching as far south as Papua New Guinea and Australia [2]. Cymbidium sinense is a perennial terrestrial species native to China and has more than a thousand years of cultural history. It holds a strong position in traditional flower markets in China, Japan, Korea, and Southeast Asia. It is of great horticultural value as an ornamental plant because of its elegance, its upright leaves, and beautiful and fragrant flowers. C. sinense blooms in winter from January to March and is usually regarded as a Spring Festival flower. Although $C$. sinense is a valuable orchid, its flowering pathway is not clearly understood. Variation in environmental conditions, including light, temperature and hormones, is likely to regulate flowering time and flower quality. Flowering regulation technology for $C$. sinense is currently scarce on the market, which seriously hinders the development of the orchid industry and also reduces the economic value of C. sinense.

The transition from vegetative growth to flowering is very importance because flowering is the first step of sexual reproduction [3]. In Arabidopsis thaliana, flower initiation takes place via four (gibberellin, autonomous, vernalization, light-dependent) genetic pathways. These processes are integrated by the function of the FLOWERING LOCUS D (FD), FLOWERING LOCUS E (FE), FLOWERING WAGENINGEN (FWA), PROTODERMAL FACTOR2 (PDF2), SUPPRESSOR OF OVEREXPRESSION OF CO 1(SOC1), and FLOWERING LOCUS T (FT) genes. The integrated signal of floral induction is transmitted to the floral meristem identity genes $L E A T Y(L F Y)$ and APETALA1 (AP1), after which floral morphogenesis takes place [4]. Currently only a few flowering genes have been cloned from orchids. DOH1 and DOMADS1 were isolated and identified from Dendrobium Madame. $D O H 1$ plays a negative regulatory role in floral formation while DOMADS1 is a marker gene specifically expressed in the shoot apical meristem during floral transition. $D O H 1$ is a possible upstream regulator of DOMADS1 and inhibits its expression [5,6]. Some MADS-box function genes were also isolated from Dendrobium crumenatum. They include APETALA2 (AP2), PISTILLATA (PI)/ GLOBOSA (GLO), APETALA3 (AP3) /DEFIENCE (DEF)like, AGAMOUS (AG) and SEPALLATA (SEP) genes, which play an important role in the early regulation of the conversion to flowers [7]. OMADS3 was isolated and characterized from Oncidium Gower Ramsey with a function similar to a functional gene regulating flower formation as well as floral initiation [8]. OMADS1 from $O$.
Gower Ramsey promoted flower transition and formation by acting as an activator for FT and SOC1 in Arabidopsis. $O M A D S 1$ was able to strongly interact with OMADS3, which influenced flower formation and floral initiation [9].

Compared with other orchids, very little research exists on the role of flowering genes in the regulation of the vegetative-to-flowering transition and flower initiation in Cymbidium. Few reports investigating the functions of flowering time genes of Cymbidium exist. Genomic resources available for the species are also scarce. Together with the nucleotide sequences obtained by NCBI searches, 60 Cymbidium expressed sequence tags (ESTs) are, however, available. Nevertheless, this genetic data is insufficient for elucidating the molecular mechanism of floral regulation in C. sinense.

In recent years, Illumina sequencing techniques have provided fascinating opportunities in life sciences and dramatically improved the efficiency of gene discovery. However, the entire transcriptome of $C$. sinense has not been sequenced. In this study, RNA-seq and digital gene expression (DGE) were performed using Illumina technology. Illumina sequencing provided comprehensive information about gene expression at the transcriptional level that could facilitate our understanding of the molecular mechanisms of $C$. sinense floral development. Such data for $C$. sinense could also be used as an important resource to investigate the flowering pathway and various other biological mechanisms in other orchid species.

\section{Results}

Illumina sequencing and sequence assembly

To obtain an overview of the $C$. sinense transcriptome, a cDNA library was generated from an equal mixture of RNA isolated from all organs, and pair end sequenced using the Illumina Hiseq 2000 platform. After cleaning and quality checks, 54 million 90-bp reads were assembled into 147162 contigs with a mean length of $326 \mathrm{bp}$ (Table 1). Using paired-end reads, these contigs were further assembled into 83580 unigenes by Trinity, including 13315 clusters and 70265 singletons, with a mean length of $612 \mathrm{bp}$. The size distribution of these

Table 1 Summary for $C$. sinense transcriptome

\begin{tabular}{ll}
\hline Total number of raw reads & $59,512,598$ \\
Total number of clean reads & $54,248,006$ \\
Total clean nucleotides (nt) & $4,882,320,540$ \\
Average read length & 90 \\
Total number of contigs & 147,162 \\
Mean length of contigs & 326 \\
Total number of unigenes & 83,580 \\
Mean length of unigenes & 612 \\
\hline
\end{tabular}


contigs and unigenes are shown in Figures 1 and 2. The assembly produced a substantial number of large contigs: 11852 contigs were $>1,000 \mathrm{bp}$ in length and 26698 contigs were $>500 \mathrm{bp}$, although most contigs were between 200 and $300 \mathrm{bp}$ in length (Figure 1). 17644 unigenes were $>1,000 \mathrm{bp}$ in length (Figure 2).

\section{Annotation of predicted proteins}

After searching the reference sequences using BLASTX against nr, SwissPort, COG and KEGG, we found a total of 41687 (49.88\% of all unigenes) unigenes providing a significant BLAST result (Table 2).

Among the 41687 unigenes, approximately $36.1 \%$ could be annotated in COG based on sequence homologies. In the COG classification, 15041 unigenes were classified into 25 function classifications (Figure 3). 'General function prediction' was dominant. 'Translation,',replication, recombination and repair' and 'posttranslational' also shared a high-percentage of genes among the categories, and only a few genes matched the terms 'nuclear structure' and 'extracellular structures'. 2199 unigenes were annotated as the 'signal transduction mechanisms' category, which suggests that our study may allow for the identification of novel genes involved in signal transduction pathways. The COG analysis showed that the identified genes are involved in various biological processes.

\section{GO classification for unigenes}

We used GO assignments to classify the functions of the predicted $C$. sinense unigenes. 16565 annotated unigenes were further categorized into 44 functional groups (Figure 4). Metabolic and cellular processes were the most highly represented groups in the biological process category. 5986 unigenes were annotated as the 'metabolic process' category, which suggests that our study may allow for the identification of novel genes involved in secondary metabolite synthesis pathways. Cell and cell parts were dominant groups in the "cellular component function" category. In the "molecular function" category, a high percentage of genes came from the 'binding' (41.1\%) and 'catalytic activity' (44.6\%) groups. In the 'nitrogen utilization,' virion' and 'translation regulator activity' groups, only a single unigene was annotated for each (Figure 4).

\section{Metabolic pathway assignment by KEGG}

In our study, 41687 annotated sequences were mapped to the reference canonical pathways in KEGG. In total, 23092 sequences were assigned to 126 KEGG pathways (Additional file 1). The pathways with most representation by the unigenes were metabolic pathways ( 5179 members, $22.43 \%$ ) and biosynthesis of secondary metabolites (2161 members, 9.36\%). These pathways provide a valuable resource for investigating specific processes, functions and pathways during C. sinense research. Interestingly, 1397 unigenes involved in plant hormone signal transduction were found, which contained 9 pathways (Table 3) controlling the signal transduction of several plant growth regulators, for example, auxin, cytokinin, gibberellin, abscisic acid, ethylene, brassinosteroid, jasmonic acid and salicylic acid. These hormones regulate seed dormancy, plant vegetative and reproductive growth, fruit ripening senescence and stress response.

The circadian clock is an important factor controlling plant physiology, and is also an important part of the photoperiod pathway. It regulates physiological activities by controlling the circadian rhythm [10]. A circadian rhythm was found in the KEGG pathway involving $222(0.97 \%)$ unigenes. The detailed metabolic pathway for the circadian rhythm is shown in Figure 5. Every gene in the pathway was associated with several



Figure 1 The length distribution of assembled $C$. sinense contigs. 




Figure 2 The length distribution of assembled C. sinense unigenes.

unigenes. The pathway will be useful for further studies on the effect of the photoperiod pathway on $C$. sinense flowering-related processes.

\section{Identifying $C$. sinense flowering time-associated genes and MADS-box genes}

According to the annotation of unigenes, we obtained 120 genes associated with flowering time. Some genes are shown in Table 4. These include photoperiod pathway genes such as GIGANTEA (GI), EARLY FLOWERING 3 (EIF3), PHYTOCHROME INTERACTING FACTOR 3 (PIF3), LATE ELONGATED HYPOCOTYL (LHY), CHALCONE SYNTHASE (CHS), CIRCADIAN CLOCK ASSOCIATED 1 (CCA1), and CO; vernalization pathway genes related to VERNALIZATION (VRN); floral integrator pathway genes related to CAULOFLOWER (CAL), AP2, FT, SOC1 and TERMINAL FLOWER 1(TFL); and floral meristem identity genes $L F Y$ and $A P 1$ were all identified in our $C$. sinense transcriptome database. Additionally, 73 MADS-box unigenes were also discovered (Additional file 2). All these unigenes are important resources for the study of floral development and flower organ formation in the future.

\section{Identifying $C$. sinense CONSTANS-like gene family} $C O$ plays a central regulatory role in the photoperiod pathway, and is regulated by the circadian clock and

Table 2 Statistics of annotation results for C. sinense unigenes

\begin{tabular}{lllllll}
\hline Sequence file & All & NR & SwissPort & KEGG & COG & GO \\
\hline JX-Unigene & 41,687 & 41,161 & 30,606 & 23,092 & 15,041 & 16,565 \\
\hline
\end{tabular}

light $[11,12]$. To determine the $C O L$ gene family of $C$. sinense, we analyzed the transcriptome database generated by this study and found 28 unigenes annotated as zinc finger protein CONSTANS. As shown in Additional file 3, they showed homology with 12 Arabidopsis COL genes.

We selected seven unigenes which have two conserved B-box domains and a CCT domain to further examine their relationship with other $C O L$ genes from Arabidopsis, rice, Phalaenopsis and $C$. sinense. The seven unigenes are shown in Table 5. Their amino acid sequences were used to construct a phylogenetic tree using MEGA4 [13] to determine genetic distances. The phylogenetic tree was showed that all members could be divided into three divergent groups (Figure 6). Unigene9883 and CL11547. Contig1 were clustered with $\mathrm{CsCO}$, PhalCOL as well as OsHd1 in group I. They are closely related to Arabidopsis AtCO/AtCOL1/AtCOL2. CL10349.Contig1, Unigene55660, CL1617.Contig1 and CL1617.Contig2 were clustered in group II. CL1617.Contig1 and CL1617.Contig2 are closely related to AtCOL9/AtCOL10. Unigene55650 is closely related to $A t C O L 13$, and CL10349.Contig1 is closely related to AtCOL14/AtCOL15. Unigene10682 was assigned to group III and is closely related to AtCOL6/AtCOL16. These results are consistent with the data shown in Table 5 .

\section{DGE library sequencing and evaluation}

The materials from VP, FDP, and RP were chosen to construct three DGE libraries, then which were sequenced. Three DGE sequencing quality evaluation and alignment statistics were shown in Table 6. The percent of all low quality reads including only adaptor and containing $\mathrm{N}$ reads was less than $0.5 \%$ in three libraries. $99.5 \%$ of raw tags in each library were clean tags. After filtering the low 


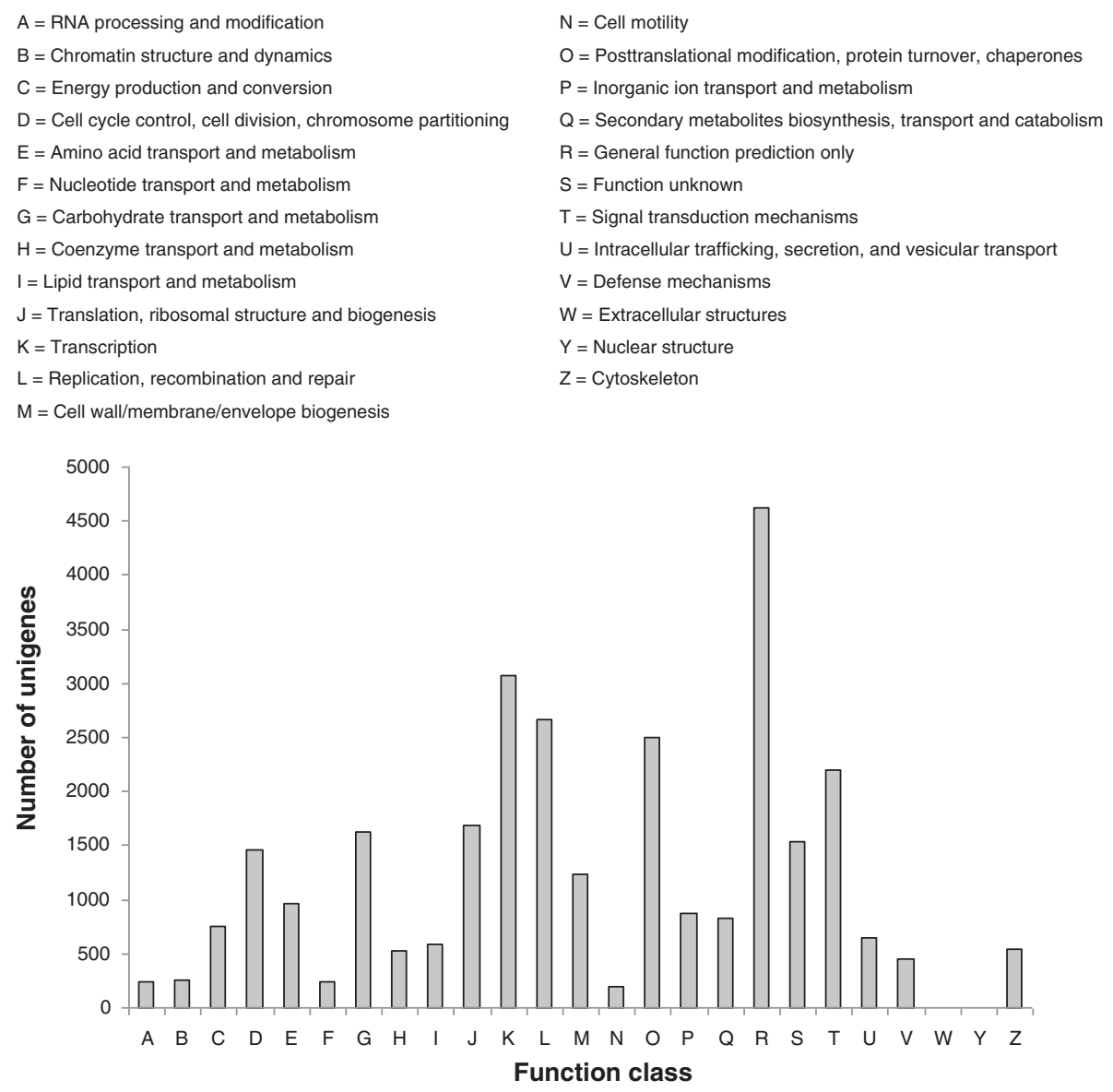

$\mathrm{N}=$ Cell motility

$\mathrm{O}=$ Posttranslational modification, protein turnover, chaperones

$\mathrm{P}=$ Inorganic ion transport and metabolism

$\mathrm{Q}=$ Secondary metabolites biosynthesis, transport and catabolism

$\mathrm{R}=$ General function prediction only

$\mathrm{S}=$ Function unknown

$\mathrm{T}=$ Signal transduction mechanisms

$U=$ Intracellular trafficking, secretion, and vesicular transport

$\mathrm{V}=$ Defense mechanisms

$W=$ Extracellular structures

$\mathrm{Y}=$ Nuclear structure

$\mathrm{Z}=$ Cytoskeleton

Function class

Figure 3 COG function classification of $C$. sinense unigenes.

quality tags, the total number of clean tags in each library was $11.98,12.65$ and 12.16 million, respectively. Among the clean tags, the number of sequences that could be mapped to transcriptome sequences amounted to 10.79 , 11.85 and 10.71 million, corresponding to $90.10,93.67$ and $88.07 \%$ in the three libraries, respectively.

\section{Variation in gene expression among three developmental stages}

To identify the differentially expressed genes during three developmental stages, the number of clean tags for each gene was calculated, and the genes that were differentially expressed between the two samples were identified according to the method described by Audic and Claverie [14].

The variations in gene expression were analyzed by comparing VP and FDP, VP and RP, and FDP and RP. The comparison between VP and FDP revealed significant variations in expression. A total of 5314 genes, including 613 up-regulated and 4701 down-regulated genes, were identified in FDP compared to VP (Figure 7). 34 genes showed specific expression in FDP and 107 genes showed specific expression in VP. These specific expression genes were listed in Additional file 4.

Based on the GO functional classification, most of the differential expression gene sets demonstrated down-regulated expression in the FDP library, and these genes were mainly correlated to membrane, vesicle and cellular component organization (Additional file 5). In the KEGG classification, 6 gene sets were significantly enriched, and most of these genes were mainly downregulated in the FDP library and correlated to plant hormone signal transduction (Additional file 5).

Meanwhile, we compared the variations in gene expression between VP and RP. A total of 14795 genes, including 14125 up-regulated and 761 down-regulated genes, were identified in RP compared to VP (Figure 7). 1408 genes showed specific expression in RP and 38 genes showed specific expression in VP. Those genes that showed specific expression were listed in Additional file 6.

Based on the GO functional classification, most of the gene sets demonstrated up-regulated expression in the RP library, and these genes were mainly correlated to hydrolase activity, binding and organelle parts 




Figure $4 \mathrm{GO}$ classification of $C$. sinense unigenes.

(Additional file 7). In the KEGG classification, 13 gene sets were significantly enriched, and most of these genes were mainly up-regulated in the FDP library and correlated to the spliceosome and ribosome (Additional file 7).

A comparison between FDP and RP revealed more variations in gene expression. The results revealed 20572 genes with significant differential expression levels between

Table 3 The pathways and the products involved in the pathway of plant hormone signal transduction

\begin{tabular}{lll}
\hline Pathway & Product & Pathway ID \\
\hline Brassinosteroid biosynthesis & Brassinosteroid & ko00905 \\
Carotenoid biosynthesis & Abscisic acid & Ko00906 \\
Cysteine and methionine metabolism & Ethylene & Ko00270 \\
Diterpenoid biosynthesis & Gibberellin & Ko00904 \\
Indole alkaloid biosynthesis & Indole-acetic acid & Ko00901 \\
a-Linolenic acid metabolism & Jasmonic acid & Ko00591 \\
Phenylalanine metabolism & Salicylic acid & Ko00360 \\
Tryptophan metabolism & Auxin & Ko00380 \\
Zeatin biosynthesis & Cytokinin & ko00908 \\
\hline
\end{tabular}

FDP and RP. Among them, 20040 and 532 genes were up-regulated and down-regulated, respectively (Figure 7). 2535 genes showed specific expression in RP and 25 genes showed specific expression in FDP. Those genes that showed specific expression are listed in Additional file 8.

Based on the GO functional classification, most of the gene sets demonstrated up-regulated expression in the RP library, and these genes were mainly correlated to binding, membrane-bounded organelles and organelle parts (Additional file 9). In the KEGG classification, 12 gene sets were significantly enriched, and most of these genes were mainly up-regulated in the RP library and correlated to the spliceosome and RNA transport (Additional file 9).

We also focused on the genes related to flower development and chose some genes that showed significantly different expression among three developmental phases (Figure 8). The functional annotation for these unigenes was listed in Additional file 10 . For example, Unigene40123 demonstrated higher expression levels in FDP and RP than in VP, Unigene33969 showed higher expression levels in RP than in VP and FDP, 




and Unigene65459 showed higher expression levels in VP and RP than in FDP, which were all homologous with Arabidopsis thaliana auxin response factor 2 (ARF2) and related to auxin metabolism. Other significant expression of different genes among the three developmental phases might be associated with circadian rhythm (Unigene4387, 33551,35813 , homolog with $F D$ ), the vernalization pathway (Unigene49193 and CL2992.Contig2, homologous with EMBRYONIC FLOWER2 (EMF2) and VERNALIZATION INSENSITIVE 3 (VIN3), respectively and so on. To validate DGE-tag profiling, 16 genes were selected randomly to examine the expression using RT-qPCR (Figure 9). The results were exhibited differential expression among three libraries and were identical to those obtained by DGE expression profiling. Thus, the data generated in this study is sufficient to be used as a tool to investigate some specific flowering genes which show comparative expression levels among different developmental phases.

\section{Discussion}

\section{Illumina sequencing and sequence annotation}

C. sinense is a very popular and traditional orchid in China. However, little is known about the mechanisms responsible for floral development and genomic information for $C$. sinense is currently unavailable. The aims of this project were to generate a large amount of cDNA sequence data that would facilitate more detailed studies in C. sinense, and to identify the genes controlling flowering time. The availability of transcriptome data for $C$. sinense will meet the initial information needs for functional studies of this species and its relatives. In this study, a combination of RNA-seq and three DGE analyses were performed using Illumina sequencing, which generated 54,248,006 high quality reads that were assembled into 83580 unigenes with an average sequence length of 612 base pairs. These unigenes were used for BLASTX and annotation against protein databases like nr, SwissPort, COG, KEGG and GO. In total, 41687 sequences were identified through BLAST searches and $50.1 \%$ unigenes had no homologues in the NCBI database. This may indicate that $C$. sinense vegetative and reproductive growth contains many unique processes and pathways.

\section{Identifying $C$. sinense flowering time-associated genes}

The photoperiod and vernalization pathways are two important genetic networks of flowering control [4]. In our study, the sequences associated with both pathways could be identified (Table 4). The photoperiod pathway comprises three parts: photoreceptors, a circadian clock and an output pathway from the clock specific to 
Table $4 \mathrm{C}$. sinense unigenes that share homology with flowering time genes

\begin{tabular}{|c|c|c|c|}
\hline Category & Gene ID & Homologous gene & $\mathrm{Nr}-\mathrm{ID}$ \\
\hline \multirow[t]{12}{*}{ Photoperiod pathway } & CL636.Contig1_JX & $\mathrm{Gl}$ & ADP92454.1 \\
\hline & CL8490.Contig1_JX & & ADP92454.1 \\
\hline & Unigene61105_JX & ELF3 & ABL11477.1 \\
\hline & Unigene61030_JX & PIF3 & NP_001063455.1 \\
\hline & Unigene31120_JX & LHY & ACF60466.1 \\
\hline & Unigene14045_JX & $\mathrm{CHS}$ & NP_001064831.1 \\
\hline & CL2407.Contig1_JX & & O23729.1 \\
\hline & Unigene68739_JX & CCA1 & ABW87009.1 \\
\hline & CL1617.Contig2_JX & $\mathrm{CO}$ & NP_001047975.1 \\
\hline & CL1617.Contig1_JX & & NP_001057441.1 \\
\hline & CL10349.Contig1_JX & & NP_001062363.2 \\
\hline & CL10838.Contig1_JX & & ADL36678.1 \\
\hline \multirow[t]{3}{*}{ Vernalization pathway } & Unigene49191_JX & VRN2 & ABD85301.1 \\
\hline & Unigene49192_JX & & ABD85301.1 \\
\hline & Unigene50102_JX & & ABD85301.1 \\
\hline \multirow[t]{23}{*}{ Floral integrator pathway } & Unigene4182_JX & FT/HD3a & ADI58462.1 \\
\hline & Unigene43540_JX & & ABX11019.1 \\
\hline & Unigene7817_JX & & CBY25182.1 \\
\hline & CL743.Contig1_JX & & ADP89470.1 \\
\hline & CL743.Contig2_JX & & ADP89470.1 \\
\hline & Unigene35813_JX & TFL & ACX53295.1 \\
\hline & Unigene36169_JX & & BAD02372.1 \\
\hline & CL11326.Contig1_JX & AP2 & ABB90554.1 \\
\hline & CL12341.Contig1_JX & & NP_001147356.1 \\
\hline & CL1488.Contig1_JX & & AAZ95247.1 \\
\hline & CL1700.Contig1_JX & & AAZ66389.1 \\
\hline & CL2122.Contig1_JX & & XP_002304138.1 \\
\hline & CL260.Contig1_JX & & XP_002274890.1 \\
\hline & CL260.Contig2_JX & & XP_002274890.1 \\
\hline & CL260.Contig3_JX & & XP_002274890.1 \\
\hline & CL260.Contig4_JX & & XP_002274890.1 \\
\hline & CL3589.Contig1_JX & & XP_002325111.1 \\
\hline & Unigene32766_JX & SOC1 & ADP06386.1 \\
\hline & Unigene59851_JX & & ADJ67241.1 \\
\hline & Unigene32196_JX & & BAC55080.1 \\
\hline & Unigene10614_JX & & ADJ67238.1 \\
\hline & Unigene59851_JX & CAL & ADJ67241.1 \\
\hline & Unigene10812_JX & & ADI58464.1 \\
\hline \multirow[t]{5}{*}{ Floral meristem identity } & Unigene32196_JX & LFY & BAC55080.1 \\
\hline & Unigene630_JX & & AAY40170.1 \\
\hline & Unigene2289_JX & AP1 & ADP00515.1 \\
\hline & CL162.Contig1_JX & & ADJ67240.1 \\
\hline & CL162.Contig2_JX & & ADJ67240.1 \\
\hline
\end{tabular}


Table 5 C. sinense unigenes that share homology with CONSTANS-like genes of Arabidopsis

\begin{tabular}{llll}
\hline Gene ID & Length of gene (bp) & Swissport annotation & Swissport-ID \\
\hline CL11547.Contig1_JX & 955 & Arabidopsis thaliana COL1 & O50055 \\
Unigene9883_JX & 1023 & Arabidopsis thaliana COL2 & Q96502 \\
Unigene10682_JX & 1636 & Arabidopsis thaliana COL6 & Q8LG76 \\
CL1617.Contig1_JX & 1957 & Arabidopsis thaliana COL9 & Q9SSE5 \\
CL1617.Contig2_JX & 2043 & Arabidopsis thaliana COL9 & Q9SSE5 \\
Unigene55650_JX & 1392 & Arabidopsis thaliana COL13 & O82256 \\
CL10349.Contig1_JX & 784 & Arabidopsis thaliana COL14 & O22800 \\
\hline
\end{tabular}

flowering [15]. Light signals are first received by two photoreceptors, phytochromes (PhyA, PhyB, PhyC, PhyD, PhyE) and cryptochromes (Cryl, Cry2) [16-20], which process the physical signals and produce a circadian clock [21-23]. The circadian clock provides a seasonal signal that adjusts plant development and regulates flowering time. It is controlled by a group of gene complexes that are composed of TOC1, CCA1 and $L H Y$ and regulate transcriptional-translational negative feedback loops [24-26]. ELF3 affects light input to the oscillator $[27,28]$. The processed signal is transmitted to the GI gene and the resultant signal activates the $C O$ gene [11]. GI is another clock-associated protein known to regulate circadian oscillation and flowering time of Arabidopsis [29,30]. The gi mutants are defective for the expression of CCA1 and $L H Y$ genes. The circadian clock controls the expression rhythm of CO through GI [31]. $C O$ is a transcription factor that promotes flowering by inducing the expression of the direct downstream genes FT [32] and SOC1 [33]. COL genes have been identified in some plant species, each of which seems to have a large family of these genes [34]. In Arabidopsis, there are 17 $C O L$ genes [35]. There are at least $16 C O L$ genes in the rice genome [34]. These COL proteins contain two B-box domains at the $\mathrm{N}$-terminus and a CCT domain at the C-terminus $[35,36]$. In this study, $28 \mathrm{C}$. sinense COL unigenes containing the conserved CCT domain were identified (Additional file 3). Seven unigenes were used to perform the phylogenetic analysis. They were classified into three conserved $C O L$ subgroups as defined previously [35]. COL proteins belonging to different subgroups are expected to perform distinct biological roles, but only several $C O L$ genes controlling flowering time have been studied in detail [34]. We presume that seven unigenes in $C$. sinense are expected to perform distinct biological roles. Sequence homologues for other genes involved in regulation of the circadian clock described above (GI, EIF3, PIF3, LHY, CHS, CCA1) could be found in our database (Table 4). Furthermore, detailed metabolic pathways for the circadian rhythm and 222 related unigenes, such as phytochromes (PhyA, PhyB, PhyC, PhyD, PhyE) and cryptochromes (Cryl, Cry2) were found
(Figure 5). Thus, these photoperiod pathways may be concerned with the regulation of flowering time in C. sinense.

VRN2 is a key gene involved in the vernalization pathway [37]. It promotes flowering by repressing the expression of FLOWERING LOCUS C (FLC) [38]. VRN2 codes for a protein with homology to PcG proteins [39]. Thus, VRN2 may function to keep the FLC-chromatin state for down-regulation. Sequence homologs for VRN2 could be found in our database (Table 4). Furthermore, temperature obviously affects the flowering time of $C$. sinense. We speculate that the vernalization pathway is related to the regulation of flowering time in C. sinense.

\section{Identifying $C$. sinense flowering integration genes and floral meristem identity genes}

Flowering integration genes accept the signal from the genetic pathway, and then induce floral meristem identity (FMI) genes for flowering as a whole [4]. At present, three integration genes, including SOC1/AGL20, FT and TFL, have been identified. SOC1 and FT are the direct target genes for $C O$. The expression of FT and SOC1 is controlled positively not only by the light pathway, but also by the autonomous pathway acting negatively through FLC [40]. The vernalization signal increases SOC1 expression, presumably by reducing FLC levels, and SOC1 can also be up-regulated by a gibberellin pathway. Accordingly, SOC1 and FT act as the convergence of all four pathways [41]. TFL1 codes for a protein with homology to FT [42]. The mutations in TFL1 are semidominant and early flowering with a determinate inflorescence [43]. Thus, TFL1 codes for a repressor of flowering. TFL2 functions as a negative repressor of FT expression. Sequence homologs for the floral integrator pathway genes related to FT, SOC1 and TFL were all identified in our $C$. sinense transcriptome database (Table 4).

The floral meristem is initiated by a set of FMI genes that include $L F Y, A P 1, C A L, A P 2$, and UNUSUAL FLORAL ORGANS (UFO) [44-47]. Among them, LFY and $A P 1$ play a central role in flower meristem identity [48-50]. LFY regulates inflorescence shape and controls 




flowering time [51,52]. AP1 is the downriver target gene of $L F Y$. $L F Y$ induces expression of AP1. LFY protein, combined with the AP1 promoter activates the transcription of $A P 1$ [53]. The phenotype of the loss-of-function-type mutations in $L F Y$ or $A P 1$ gene is as follows: Flowers either have vegetative characteristics or have been replaced by vegetative shoots [54]. The phenotype of double mutations in lfy/ap1 is a scarce floral structure [49,55], which elucidated the functional
Table 6 DGE sequencing quality evaluation and alignment statistics

\begin{tabular}{|c|c|c|c|}
\hline Summary & VP & FDP & $\mathrm{RP}$ \\
\hline Only adaptor reads & 25804 & 19835 & 37799 \\
\hline Only adaptor reads\% & $0.21 \%$ & $0.16 \%$ & $0.31 \%$ \\
\hline Containing $\mathrm{N}$ reads & 37 & 40 & 43 \\
\hline Containing $\mathrm{N}$ reads $\%$ & $0.00 \%$ & $0.00 \%$ & $0.00 \%$ \\
\hline Low quality reads & 25686 & 32107 & 16577 \\
\hline Low quality reads $\%$ & $0.21 \%$ & $0.25 \%$ & $0.14 \%$ \\
\hline Total clean reads & 11981512 & 12647899 & 12164959 \\
\hline Total clean reads $\%$ & $99.57 \%$ & $99.59 \%$ & $99.55 \%$ \\
\hline Total base pairs & 587094088 & 619747051 & 596082991 \\
\hline Total mapped reads to gene & 10795616 & 11847282 & 10713910 \\
\hline Total\% of mapped reads & $90.10 \%$ & $93.67 \%$ & $88.07 \%$ \\
\hline Perfect match & 7558927 & 10028451 & 8649669 \\
\hline Perfect match\% & $63.09 \%$ & $79.29 \%$ & $71.10 \%$ \\
\hline$\leq 2$ bp mismatch & 3236689 & 1818831 & 2064241 \\
\hline$\leq 2$ bp mismatch\% & $27.01 \%$ & $14.38 \%$ & $16.97 \%$ \\
\hline Unique match & 9389031 & 10763499 & 8207609 \\
\hline Unique match\% & $78.36 \%$ & $85.10 \%$ & $67.47 \%$ \\
\hline Multi-position match & 1406585 & 1083783 & 2506301 \\
\hline Multi-position match\% & $11.74 \%$ & $8.57 \%$ & $20.60 \%$ \\
\hline Total unmapped reads & 1185896 & 800617 & 1451049 \\
\hline Total unmapped reads\% & $9.90 \%$ & $6.33 \%$ & $11.93 \%$ \\
\hline
\end{tabular}

redundancies in $A P 1-L F Y$. Functional redundancies were detected in AP1-CAL, AP2-AP1 and AP2-LFY genes [56,57]. $A P 1$ and $C A L$ belong to the MADS domain genes and $U F O$ codes for an F-box protein which degrades other proteins through a ubiquitation pathway $[46,58,59] . L F Y$ is also thought to promote $C A L$ and $U F O$ expression $[60,61]$. Therefore, these FMI genes cooperate together to

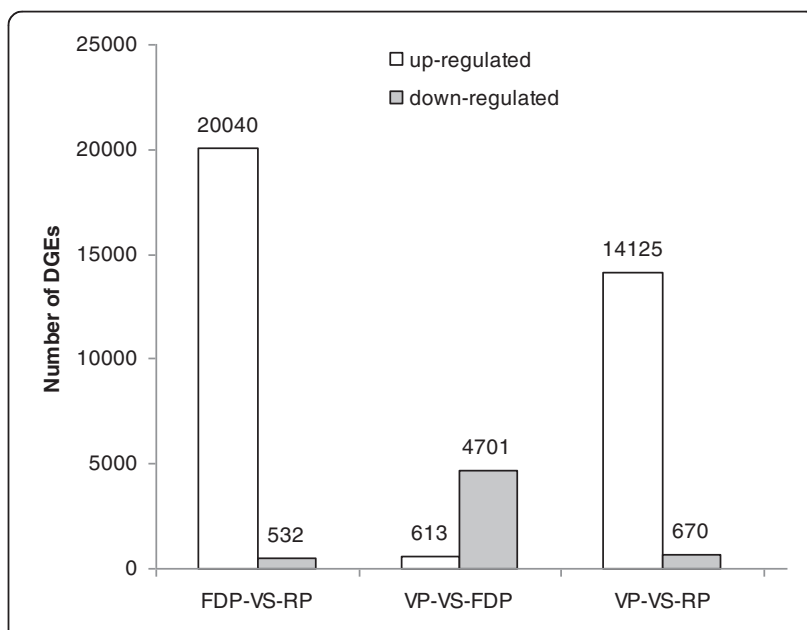

Figure 7 Numbers of DGE unigenes in each comparison. 




Figure 8 Differential expression genes related with flower development in C. sinense DGE. Each column represents an experimental sample (e.g. VP, FDP and RP) and each row represents a gene. Expression differences are shown in different colors. Red means high expression and green means low expression.

promote the transition from vegetative growth to reproductive growth. Sequence homologs for $F M I$ genes $C A L, A P 2, L F Y$ and $A P 1$ were all identified in our $C$. sinense transcriptome database. These information of flowering integration genes and FMI genes would facilitate more detailed studies on the mechanism of floral differentiation for C. sinense.

\section{Differential expression of genes among three developmental phases}

We have identified the putative homologs of $C O L$ and other key genes involved in controlling flowering time in $C$. sinense. More studies on their expression patterns and on their interactive relationships in the future could be used to elucidate the molecular mechanisms that regulate the floral transition and the flowering genetic pathway in C. sinense. To better understand the information related to gene expression obtained for $C$. sinense, we created three DEG libraries to analyze the gene expression patterns under three developmental phases. In the comparison between FDP with VP, most of the expressed genes were downregulated and parts were up-regulated. In contrast, when RP was compared to VP and FDP, most of the expressed genes were up-regulated (Figure 7). Furthermore, a large amount of genes which showed specific expression in the three phases were likely involved in floral development. 



Figure 9 The qRT-PCR analysis of gene expression in VP (blue bars), FDP (red bars) and RP (green bars). The $y$-axis indicates fold change in expression among the samples VP, FDP, and RP using the results from RT-qPCR. The Lg(Relative Quantitation) of 16 genes in the VP was calibrated as zero. 

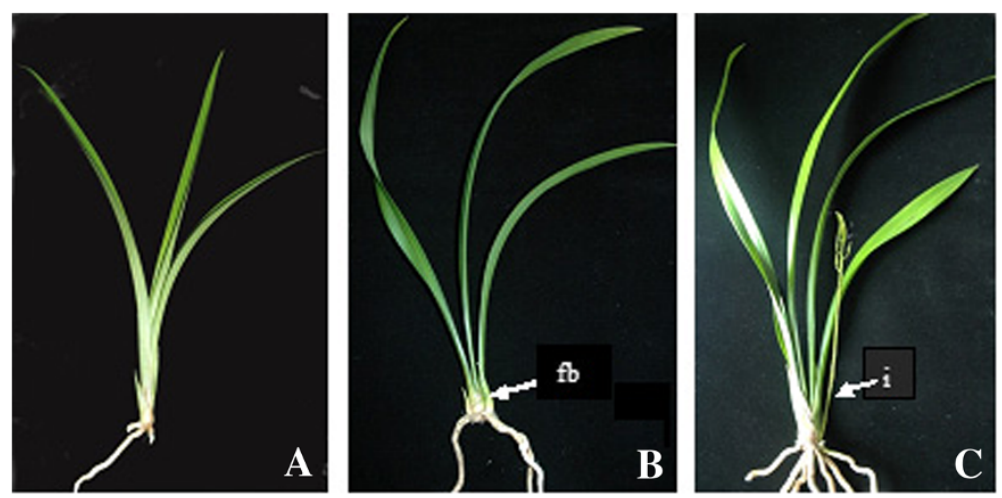

Figure 10 Organs from $C$. sinense used to prepare DGE libraries for Illumine sequencing. (A) A young C. sinense plant in the vegetative phase (VP); (B) A mature C. sinense plant with floral bud (fb) in the floral differentiation phase (FDP); (C) A mature $C$. sinense plant with inflorescence (i) in the reproductive phase (RP).

When FDP was compared to VP, TFL1 showed specific expression in VP. TFL1 codes for a flowering repressor that can function to control inflorescence architecture and extend the phases of shoot meristems in Arabidopsis [40]. Our sequence was consistent with this theory. Among all specific expression of annotated genes in RP compared to VP or FDP, auxin response factors (ARF2) were identified in RP. Auxin signaling is important in the regulation of developmental transitions such as seed germination, induction of flowering, leaf senescence and shedding of senescent organs. ARFs are transcription factors that mediate responses to the plant hormone auxin [62]. For example, ARF2 promotes the transition between multiple stages of Arabidopsis development, including initiation of flowering, rosette leaf senescence, floral organ abscission and silique ripening. In contrast, ARF1 is a transcriptional repressor and the arf1 mutation increases the transcription of Aux/IAA genes in Arabidopsis flowers [63]. Moreover, most of the specific gene expression was not annotated as a relative function, which could assist in the search for some new genes associated with floral development.

Although the molecular functions of $C$. sinense genes and the associated floral genetic pathways remain unknown, the present transcriptome analysis provides valuable information regarding $C$. sinense development, which could facilitate further investigations of the detailed floral development mechanisms of this culturally important orchid.

\section{Conclusion}

The combination of RNA-seq and DGE analysis based on Illumina sequencing technology provided comprehensive information on gene expression. Candidate genes on flowering time genes, $M A D S$-box genes and $C O L$ genes were rapidly identified by this approach. This data could be used as a tool to investigate the flowering pathway and various other biological pathways in $C$. sinense.

\section{Methods}

Plant materials and growth conditions

C. sinense 'Qi Jian Bai Mo' plants used in this study were grown and maintained in pots in a greenhouse of the South China Botanical Garden at a day/night temperature of $28 / 25^{\circ} \mathrm{C}$ with a $12-\mathrm{h}$ period. The cDNA libraries were prepared from the entire plant of $C$. sinense at vegetative and reproductive stages. The vegetative phase (VP) samples were collected from a non-pseudobulb shoot (Figure 10A). The floral differentiation phase (FDP) samples were collected from the entire adult plant which had developed floral buds at the base of the pseudobulb (Figure 10B). The reproductive phase (RP) samples were collected from entire adult plants which had developed a peduncle with floral organs at the base of the pseudobulb (Figure 10C).

Fresh samples were used to extract total RNA immediately.

\section{cDNA library preparation and Illumina sequencing for transcriptome analysis}

Total RNA was extracted using Column Plant RNAout2.0 (Tiandz, Inc. Beijing, China) according to the manufacturer's protocol. To obtain complete gene expression information, a pooled RNA sample including roots, leaves, pseudobulbs, flower buds, young and mature inflorescences was used for transcriptome sequencing analysis. According to the Illumina manufacturer's instructions, poly $(\mathrm{A})^{+}$RNA was purified from $20 \mathrm{mg}$ of pooled total RNA using oligo(dT) magnetic beads. Fragmentation buffer was added to interrupt mRNA to short fragments. Using these short fragments as templates, a random hexamer-primer was used to synthesize the first-strand cDNA. Second-strand cDNA 
was synthesized using 10×buffer, $25 \mathrm{mM}$ dNTPs, 20-60 $\mathrm{U} / \mu \mathrm{l}$ RNaseH and $5 \mathrm{U} / \mu \mathrm{l}$ DNA polymerase I. Short fragments were purified with the QiaQuick PCR extraction kit and resolved with EB buffer for end reparation and for adding poly(A). Thereafter, the short fragments were connected with sequencing adapters. After agarose gel electrophoresis, suitable fragments were selected for PCR amplification as templates. Finally, the library was sequenced using Illumina $\mathrm{HiSeq}^{\mathrm{TM}}$ 2000. All raw transcriptome data were deposited in the GeneBank Short Read Archive (Accession SRA058042).

\section{Analysis of Illumina sequencing results}

Raw reads from the image data output from the sequencing machine were generated by base calling. After filtering raw reads, de novo assembly of the transcriptome was carried out with a short reads assembling program - Trinity [64]. Trinity connects the contigs and obtains sequences defined as unigenes.

The generated unigenes were used for BLASTX and annotation against protein databases, including non-redundant (nr), SwissPort, COG, KEGG, and GO protein database, with a cut-off E-value of 0.00001. GO (http://www.geneontology.org) has three ontologies: molecular function, cellular component and biological process. With $\mathrm{nr}$ annotation, we used the Blast2GO program [65] to obtain the GO annotation of unigenes. After obtaining the GO annotation for every unigene, we used WEGO software [66] to perform GO functional classification for all unigenes and to understand the distribution of gene functions. KEGG is a major public pathway-related database [67] that is able to analyze a gene product during a metabolic process and related gene function in cellular processes. With the help of the KEGG database, we can further study genes' biological complex behaviors, and by KEGG annotation we can obtain pathway annotation for unigenes. The KEGG pathways annotation was performed using Blastall software against the KEGG database.

\section{Digital gene expression (DGE) library preparation and sequencing}

Total RNA was extracted from VP, FDP and RP using Column Plant RNAout2.0. mRNA was enriched by using oligo(dT) magnetic beads. After adding the fragmentation buffer, the mRNA was interrupted to short fragments (about $200 \mathrm{bp}$ ). Then the first strand cDNA was synthesized by a random hexamer-primer using the mRNA fragments as templates. 10xbuffer, $25 \mathrm{mM}$ dNTPs, 20-60 $\mathrm{U} / \mu \mathrm{l} \mathrm{RNaseH}$ and $5 \mathrm{U} / \mu \mathrm{l}$ DNA polymerase I were added to synthesize the second strand. The double-stranded cDNA was purified with a QiaQuick PCR extraction kit and washed with EB buffer for end repair and single nucleotide A (adenine) addition. Finally, sequencing adaptors were ligated to the fragments. The required fragments were purified by agarose gel electrophoresis and enriched by PCR amplification. The library products were ready for sequencing analysis via Illumina $\mathrm{HiSeq}^{\mathrm{TM}}$ 2000. Three raw DGE data (VP, FDP and RP) were deposited in the GeneBank Short Read Archive (accession SRA058974, SRA058977, SRA058978, respectively).

\section{Analysis and mapping of DGE tags}

To map the DGE tags, the sequenced raw data were filtered to remove low quality tags (tags with an unknown nucleotide "N"), empty tags (no tag sequence between the adaptors) and tags with only one copy number (which might result from sequencing errors). For the annotation of tags, clean tags containing CATG and 21-bp tag sequences were mapped to our transcriptome reference database using SOAPaligner/soap2 [68]. Mismatches of no more than two bases were allowed in the alignment.

\section{Screening of differentially expressed genes (DEGs)}

To compare the differences in gene expression at different developmental stages, the tag frequency in the different DGE libraries was statistically analyzed according to the method described by Audic and Claverie [14]. The false discovery rate (FDR) was used to determine the threshold P-value in multiple tests. We used FDR $<0.001$ and an absolute value of the $\log _{2}$ ratio $>1$ as the threshold to determine the significant difference in gene expression. The differentially expressed genes were used for GO and KEGG enrichment analyses according to a method similar to that described by Xue [69]. GO terms, which take the corrected P-value $\leq 0.05$ as a threshold, are significantly enriched in DEGs. KEGG pathways with a Q-value $\leq 0.05$ are significantly enriched in DEGs.

Genes with similar expression patterns usually mean functional correlation. We perform cluster analysis of gene expression patterns with cluster [70] software and Java Treeview [71] software. In Figure 8, each column represents an experimental sample (e.g. VP, FDP and $\mathrm{RP}$ ), each row represents a gene. Expression differences are shown in different colors. Red means high expression and green means low expression.

\section{Quantitative real-time PCR (qRT-PCR) validation}

Total RNA was extracted as described for the DGE library preparation and sequencing. Each RNA sample was treated with RNase-free DNase (Promega) following the manufacturer's protocol in an effort to remove any residual genomic DNA (gDNA). DNase-treated RNA (2 mg) was subjected to reverse transcriptase reactions using oligo-dT primer and PrimeScript ${ }^{{ }^{\mathrm{M}}}$ Reverse Transcriptase (Takara) according to the manufacturer's protocol. 
The sequences of the specific primer sets are listed in Additional file 11. The actin gene of C. sinense (ACC NO. GU181353) was used as an internal gene. qRT-PCR was performed using the SYBR Premix Ex Taq Kit (TaKaRa) according to the manufacturer's protocol. The results were normalized to the expression level of the constitutive actin gene. A relative quantitative method (DDCt) was used to evaluate the quantitative variation.

\section{Additional files}

Additional file 1: Metabolic pathway analysis result for unigenes by KEGG annotation.

Additional file 2: The unigenes that share homology with MADS-box genes.

Additional file 3: The unigenes that share homology to CONSTANS-like genes.

Additional file 4: Specific expression of genes in the comparison between FDP with VP.

Additional file 5: Gene set enrichment analysis in the comparison between FDP with VP by GO and KEGG.

Additional file 6: Specific expression of genes in the comparison between RP and VP.

Additional file 7: Gene set enrichment analysis in the comparison between RP with VP by GO and KEGG.

Additional file 8: Specific expression of genes in the comparison between RP and FDP.

Additional file 9: Gene set enrichment analysis in the comparison between RP and FDP by GO and KEGG.

Additional file 10: The functional annotation for the unigenes related with flower development in C. sinense DGE.

Additional file 11: Primer sequences for qRT-PCR.

\section{Abbreviations}

DGE: Digital gene expression; GO: Gene ontology; KEGG: Kyoto encyclopedia of genes and genomes; AP1: Apetala1; AP2: Apetala2; AP3: Apetala3; AG: Agamous; CAL: Cauloflower; CCA1: Circadian clock associated 1; CHS: Chalcone synthase; CO: Constans; COL: Constans-like; DEF: Deficiens; ELF3: Early flowering 3; EST: Expressed sequence tag; EMF2: Embryonic flower 2; FD: Flowering locus D; FE: Flowering locus E; FT: Flowering locus T; FLC: Flowering locus C; FWA: Flowering wageningen; Gl: Gigantea; GLO: Globosa; LHY: Late elongated hypocotyl; LFY: Leafy; PDF2: Protodermal factor2; PIF3: Phytochrome interacting factor 3; PI: Pistillata; SEP: Sepallata; SOC1: Suppressor of overexpression of co 1; TFL1: Terminal flower 1; TOC1: Timing of cab expression 1; VIN3: Vernalization insensitive 3; VRN1: Vernalization 1; UFO: Unusual floral organs.

\section{Competing interests}

The authors declare that they have no competing interest.

\section{Authors' contributions}

JZ carried out the experiments, performed the bioinformatics analyses, and drafted the manuscript. KW cultured and provided the experimental material. SZ participated in the qRT-PCR experiment. JATdS critically evaluated the protocol, the data and interpretation and revised the manuscript. HX performed the bioinformatics analyses. JD designed the study and revised the manuscript. XZ and CT participated in the design and coordination. All authors read and approved the final manuscript.

\section{Acknowledgments}

This study was supported by the National Natural Science Foundation of China (CNSF31101572 and CNSF31170654), the Science and Technology
Project Foundation of Guangdong Province (2011A020201001 and 2012B091100255), Knowledge Innovation Program of the Chinese Academy Sciences (Y021121001) and the Foundation of Key Laboratory of Plant Resources Conservation and Sustainable Utilization, South China Botanical Garden, Chinese Academy of Sciences (201222ZS).

\section{Author details}

'Key Laboratory of South China Agricultural Plant Genetics and Breeding, South China Botanical Garden, The Chinese Academy of Sciences, Guangzhou 510650, China. ${ }^{2}$ Key Laboratory of Plant Resources Conservation and Sustainable Utilization, South China Botanical Garden, Chinese Academy of Sciences, Guangzhou 510650, China. ${ }^{3}$ Faculty of Agriculture and Graduate School of Agriculture, Kagawa University, Miki-cho, Kagawa 761-0795, Japan. ${ }^{4}$ Guangdong Key Laboratory for Innovative Development and Utilization of Forest Plant Germplasm, South China Agricultural University, Guangzhou 510642, China. ${ }^{5}$ School of Life Sciences, Guangzhou University, Guangzhou 510006, China. ${ }^{6}$ Guangzhou Genedenovo Biotechnology Co.,Ltd, Guangzhou 510006, China.

Received: 31 October 2012 Accepted: 15 April 2013

Published: 24 April 2013

\section{References}

1. Leitch IJ, Kahandawala I, Suda J, Hanson L, Ingrouille MJ, Chase MW, Fay MF: Genome size diversity in orchids: consequences and evolution. Ann Bot 2009, 104(3):469-481.

2. Wu ZY, Raven PH, Hong DY: Flora of China. Volume 25 (Orchidaceae). Beijing, St. Louis: Science Press and Missouri Botanical Garden Press; 2009:260.

3. Bernier G, Havelange A, Houssa C, Petitjean A, Lejeune P: Physiological signals that induce flowering. Plant Cell 1993, 5:1147-1155.

4. Komeda Y: Genetic regulation of time to flower in Arabidopsis thaliana. Annu Rev Plant Biol 2004, 55:521-535.

5. Yu H, Yang SH, Goh CJ: DOH1, a class 1 knox gene, is required for maintenance of the basic plant architecture and floral transition in orchid. Plant Cell 2000, 12(11):2143-2159.

6. Yu H, Yang SH, Goh CJ: Spatial and temporal expression of the orchid floral homeotic gene DOMADS1 is mediated by its upstream regulatory regions. Plant Mol Biol 2002, 49(2):225-237.

7. $X u$ Y, Teo LL, Zhou J, Kumar PP, Yu H: Floral organ identity genes in the orchid Dendrobium crumenatum. Plant J 2006, 46:54-68.

8. Hsu HF, Yang CH: An orchid (Oncidium Gower Ramsey) AP3-like MADS gene regulates floral formation and initiation. Plant Cell Physiol 2002, 43(10):1198-1209.

9. Hsu HF, Huang $\mathrm{CH}$, Chou LT, Yang CH: Ectopic expression of an orchid (Oncidium Gower Ramsey) AGL6-like gene promotes flowering by activating flowering time genes in Arabidopsis thaliana. Plant Cell Physiol 2003, 44(8):783-794.

10. Niwa Y, Yamashino T, Mizuno T: The circadian clock regulates the photoperiodic response of hypocotyl elongation through a coincidence mechanism in Arabidopsis thaliana. Plant Cell Physiol 2009, 50(4):838-854.

11. Putterill J, Robson F, Lee K, Simon R, Coupland G: The CONSTANS gene of Arabidopsis promotes flowering and encodes a protein showing similarities to zinc finger transcription factors. Cell 1995, 80:847-857.

12. Suarez-Lopez P, Wheatley K, Robson F, Onouchi H, Valverde F, Coupland G: CONSTANS mediates between the circadian clock and the control of flowering in Arabidopsis. Nature 2001, 410:1116-1120.

13. Tamura K, Dudley J, Nei M, Kumar S: MEGA4: molecular evolutionary genetics analysis (MEGA) software version 4.0. Mol Biol Evol 2007, 24:1596-1599.

14. Audic S, Claverie JM: The significance of digital gene expression profiles. Genome Res 1997, 7(10):986-95.

15. Simpson GG: Evolution of flowering in response to day length flipping the CONSTANS switch. Bioessays 2003, 25:829-832.

16. Ahmad M, Cashmore AR: HY4 gene of $A$. thaliana encodes a protein with characteristics of a blue-light photoreceptor. Nature 1993, 366:162-166.

17. Briggs WR, Beck CF, Cashmore AR, Christie JM, Hughes J, Jarillo JA, Kagawa T, Kanegae H, Liscum E, Nagatani A, Okada K, Salomon M, Rüdiger, Sakai T, Takano M, Wada M, Watson JC: The phototropin family of photoreceptors. Plant Cell 2001, 13:993-997.

18. Lin C, Yang H, Guo H, Mockler T, Chen J, Cashmore AR: Enhancement of the blue-light sensitivity of Arabidopsis young seedlings by a blue-light receptor cry2. Proc Natl Acad Sci USA 1998, 95:2686-2690. 
19. Parks BM: Quail PH: hy8, a new class of Arabidopsis long hypocotyl mutants deficient in functional phytochrome A. Plant Cell 1993, 5:39-48.

20. Reed JW, Nagpal P, Poole DS, Furuya M, Chory J: Mutations in the gene for the red/far-red light receptor phytochrome B alter cell elongation and physiological responses throughout Arabidopsis development. Plant Cell 1993, 5:147-157.

21. Fowler S, Lee K, Onouchi H, Samach A, Richardson K, Morris B, Coupland G, Putterill J: GIGANTEA: a circadian clock controlled gene that regulates photoperiodic flowering in Arabidopsis and encodes a protein with several possible membrane spanning domains. EMBO J 1999, 18:4679-4688.

22. Hicks KA, Albertson TM, Wagner DR: EARLY FLOWERING3 encodes a novel protein that regulates circadian clock function and flowering in Arabidopsis. Plant Cell 2001, 13:1281-1292.

23. Simpson GG, Dean C: Arabidopsis, the Rosetta stone of flowering time? Science 2002, 296:285-289.

24. Somers DE: The physiology and molecular bases of the plant circadian clock. Plant Physiol 1999, 121:9-20.

25. Alabadid D, Oyama T, Yanovsky MJ, Harmon FG, Mas P, Kay SA: Reciprocal regulation between TOC1 and LHY/CCA1 within the Arabidopsis circadian clock. Science 2001, 293:880-883.

26. Millar AJ: Input signals to the plant circadian clock. J Exp Bot 2004, 55:277-283

27. McWatters HG, Bastow RM, Hall A, Millar AJ: The ELF3 zeitnehmer regulates light signalling to the circadian clock. Nature 2000, 408:716-720.

28. Dixon LE, Knox K, Kozma-Bognar L, Southern MM, Pokhilko A, Millar AJ: Temporal repression of core circadian genes is mediated through EARLY FLOWERING 3 in Arabidopsis. Curr Biol 2011, 21:120-125.

29. Mizoguchi T, Wright L, Fujiwara S, Cremer F, Lee K, Onouchi H, Mouradov A, Fowler S, Kamada H, Putterill J, Coupland G: Distinct roles of GIGANTEA in promoting flowering and regulating circadian rhythms in Arabidopsis. Plant Cell 2005, 17:2255-2270.

30. Locke JCW, Kozma-Bognar L, Gould PD, Fehér B, Kevei E, Nagy F, Turner MS, Hall A, Millar AJ: Experimental validation of a predicted feedback loop in the multi-oscillator clock of Arabidopsis thaliana. Mol Syst Biol 2006, 2:59.

31. Doyle MR, Davis SJ, Bastow RM, McWatters HG, Kozma-Bognar L, Nagy E, Millar AJ, Amasino RM: The ELF4 gene controls circadian rhythms and flowering time in Arabidopsis thaliana. Nature 2002, 419:74-77.

32. Kardailsky I, Shukla VK, Ahn JH, Dagenais N, Christensen SK, Nguyen JT, Chory J, Harrison MJ, Weigel D: Activation tagging of the floral inducer FT. Science 1999, 286:1962-1965.

33. Samach A, Onouchi H, Gold SE, Ditta GS, Schwarz-Sommer Z, Yanofsky MF, Coupland G: Distinct roles of CONSTANS target genes in reproductive development of Arabidopsis. Science 2000, 288:1613-1616.

34. Griffths S, Dunford RP, Coupland G, Laurie DA: The evolution of CONSTANS-like gene families in barley, rice, and Arabidopsis. Plant Physiol 2003, 131:1855-1867.

35. Robson F, Costa MMR, Hepworth SR, Vizir I, Pineiro M, Reeves PH, Putterill J, Coupland G: Functional importance of conserved domains in the flowering-time gene CONSTANS demonstrated by analysis of mutant alleles and transgenic plants. Plant J 2001, 28:619-631.

36. Ledger S, Strayer C, Ashton F, Kay SA, Putterill J: Analysis of the function of two circadian-regulated CONSTANS-LIKE genes. Plant J 2001, 26:15-22.

37. Chandler J, Wilson A, Dean C: Arabidopsis mutants showing an altered response to vernalization. Plant J 1996, 10:637-644.

38. Sheldon CC, Rouse DT, Finnegan EJ, Peacock WJ, Dennis ES: The molecular basis of vernalization: The central role of FLOWERING LOCUS C (FLC). Proc Natl Acad Sci USA 2000, 97:3753-3758.

39. Gendall AR, Levy YY, Wilson A, Dean C: The VERNALIZATION 2 gene mediates the epigenetic regulation of vernalization in Arabidopsis. Cell 2001, 107:525-535.

40. Amador V, Monte E, Garcia-Martinez JL, Prat S: Gibberellins signal nuclear import of PHOR1, a photoperiod-responsive protein with homology to Drosophila armadillo. Cell 2001, 106(3):343-354.

41. Hepworth SR, Valverde F, Ravenscroft D, Mouradov A, Coupland G: Antagonistic regulation of flowering-time gene SOC1 by CONSTANS and FLC via separate promoter motifs. EMBO J 2002, 21:4327-4337.

42. Bradley D, Ratcliffe O, Vincent C, Carpenter R, Coen E: Inflorescence commitment and architecture in Arabidopsis. Science 1997, 275:80-83.

43. Alvarez J, Guli CL, Yu XH, Smyth DR: Terminal flower: a gene affecting inflorescence development in Arabidopsis thaliana. Plant J 1992, 2:103-116.
44. Mandel MA, Gustafson-Brown C, Savidge B, Yanofsky MF: Molecular characterization of the Arabidopsis floral homeotic gene APETALA1. Nature 1992, 360:273-277.

45. Jofuku KD, den Boer BGW, Van Montagu M, Okamuro JK: Control of Arabidopsis flower and seed development by homeotic gene APETALA2. Plant Cell 1994, 6:1211-1225.

46. Kempin SA, Savidge B, Yanofsky MF: Molecular basis of the cauliflower phenotype of Arabidopsis. Science 1994, 267:522-525.

47. Yanofsky MF: Floral meristems to floral organs: genes controlling early events in Arabidopsis flower development. Annu Rev Plant Physiol Plant Mol Biol 1995, 46:167-188.

48. Schultz EA, Haughn GW: $L E A F Y$, a homeotic gene that regulates inflorescence development in Arabidopsis. Plant Cell 1991, 3:771-781.

49. Weigel D, Alvarez J, Smyth DR, Yanofsky MF, Meyerowitz EM: LEAFY controls floral meristem identity in Arabidopsis. Cell 1992, 69(5):843-859.

50. Pidkowich MS, Klenz JE, Haughn GW: The making of a flower: control of floral meristem identity in Arabidopsis. Plant Sci 1999, 4:64-70.

51. Coen ES, Romero JM, Doyle S, Elliott R, Murphy G, Carpenter R: floricaula: a homeotic gene required for flower development in Antirrhinum majus. Cell 1990, 63:1311-1322.

52. Nilsson O, Lee $Y$, Blazquez MA, Weigel D: Flowering time genes modulate the response to LEAFY activity. Genetics 1998, 150:403-410.

53. Parcy F, Nilsson O, Busch MA, Lee I, Weigel D: A genetic framework for floral patterning. Nature 1998, 395:561-566.

54. Irish VF, Sussex IM: Function of the APETALA1 gene during Arabidopsis floral development. Plant Cell 1990, 2:741-753.

55. Huala E, Sussex IM: LEAFY interacts with floral homeotic genes to regulate Arabidopsis floral development. Plant Cell 1992, 4:901-913.

56. Bowman JL, Alvarez J, Weigel D, Meyerowitz EM, Smyth DR: Control of flower development in Arabidopsis thaliana by APETALA1 and interacting genes. Development 1993, 119:721-743.

57. Shannon S, Meeks-Wagner DR: Genetic interactions that regulate inflorescence development in Arabidopsis. Plant Cell 1993, 5:639-655.

58. Mandel MA, Yanofsky MF: A gene triggering flower formation in Arabidopsis. Nature 1995, 377:522-524.

59. Bai C, Sen P, Hofmann K, Ma L, Goebl M, Harper JW, Elledge SJ: SKP1 connects cell cycle regulators to the ubiquitin proteolysis machinery through a novel motif, the F-box. Cell 1996, 86:263-274.

60. Ferrandiz C, Gu Q, Martienssen R, Yanofsky MF: Redundant regulation of meristem identity and plant architecture by FRUITFULL, APETALA1 and CAULIFLOWER. Development 2000, 127:725-734.

61. Lee I, Wolfe DS, Nilsson O, Weigel D: A LEAFY co-regulator encoded by UNUSUAL FLORAL ORGANS. Curr Biol 1997, 7:95-104.

62. Hagen G, Guilfoyle TJ: Auxin-responsive gene expression: genes, promoters and regulatory factors. Plant Mol Biol 2002, 49:373-385.

63. Ellis CM, Nagpal P, Young JC, Hagen G, Guilfoyle TJ, Reed JW: AUXIN RESPONSE FACTOR1 and AUXIN RESPONSE FACTOR2 regulate senescence and floral organ abscission in Arabidopsis thaliana. Development 2005, 132:4563-4574.

64. Grabherr MG, Haas BJ, Yassour M, Levin JZ, Thompson DA, Amit I, Adiconis Z, Fan L, Raychowdhury R, Zeng Q, Chen Z, Mauceli E, Hacohen N, Gnirke A, Rhind N, di Palma F, Birren BW, Nusbaum C, Lindblad-Toh K, Friedman N, Regev A: Full-length transcriptome assembly from RNA-Seq data without a reference genome. Nat Biotechnol 2011, 29:644-652.

65. Conesa A, Gotz S, Garcia-Gomez JM, Terol J, Talon M, Robles M: Blast2GO: a universal tool for annotation, visualization and analysis in functional genomics research. Bioinformatics 2005 21(18):3674-3676

66. Ye J, Fang L, Zheng H, Zhang Y, Chen J, Zhang Z, Wang J, Li S, Li R, Bolund L, Wang J: WEGO: a web tool for plotting GO annotations. Nucleic Acids Res 2006, 34(Web Server issue):W293-297.

67. Kanehisa M, Araki M, Goto S, Hattori M, Hirakawa M, Itoh M, Katayama T, Kawashima S, Okuda S, Tokimatsu T, Yamanishi Y: KEGG for linking genomes to life and the environment. Nucleic Acids Res 2008, 36(Database issue):D480-484.

68. Li R, Yu C, Li Y, Lam TW, Yiu SM, Kristiansen K, Wang J: SOAP2: an improved ultrafast tool for short read alignment. Bioinformatics 2009, 25(15):1966-1967. 
69. Xue J, Bao YY, Li BL, Cheng YB, Peng ZY, Liu H, Xu HJ, Zhu ZR, Lou YG, Cheng JA, Zhang CX: Transcriptome analysis of the brown planthopper Nilaparvata lugens. PLoS One 2010, 5(12):e14233.

70. de Hoon MJL, Imoto S, Nolan J, Miyano S: Open Source Clustering Software. Bioinformatics 2004, 20(9):1453-1454.

71. Saldanha AJ: Java Treeview-extensible visualization of microarray data. Bioinformatics 2004, 20(17):3246-3248.

doi:10.1186/1471-2164-14-279

Cite this article as: Zhang et al.: Transcriptome analysis of Cymbidium

sinense and its application to the identification of genes associated with floral development. BMC Genomics 2013 14:279.

\section{Submit your next manuscript to BioMed Central and take full advantage of:}

- Convenient online submission

- Thorough peer review

- No space constraints or color figure charges

- Immediate publication on acceptance

- Inclusion in PubMed, CAS, Scopus and Google Scholar

- Research which is freely available for redistribution 